\title{
Erratum to: Kilotesla Magnetic Assisted Fast Laser Ignited Boron-11 Hydrogen Fusion with Nonlinear Force Driven Ultrahigh Accelerated Plasma Blocks
}

\author{
P. Lalousis $\cdot$ S. Moustaizis $\cdot$ H. Hora $\cdot$ \\ G. H. Miley
}

Published online: 26 October 2014

(c) Springer Science+Business Media New York 2014

\section{Erratum to: J. Fusion energy}

DOI 10.1007/s10894-014-9759-5

In the original version of this article, Figs. 2, 3, 4, 5, 7 and 8 need a correction due to an improvement of the computer code. The correct figures and captions are given below. There is no change in the contents of the original version.

The online version of the original article can be found under doi:10.1007/s10894-014-9759-5.

\section{P. Lalousis}

Institute of Electronic Structure and Lasers FORTH, Heraklion,

Greece

\section{S. Moustaizis}

Technical University of Crete, Chania, Greece

H. Hora $(\square)$

Department of Theoretical Physics, University of New South

Wales, Sydney, NSW, Australia

e-mail: h.hora@unsw.edu.au

\section{G. H. Miley}

Department of Nuclear, Plasma and Radiological Engineering, University of Illinois, Urbana-Champaign Campus, Urbana, IL, USA

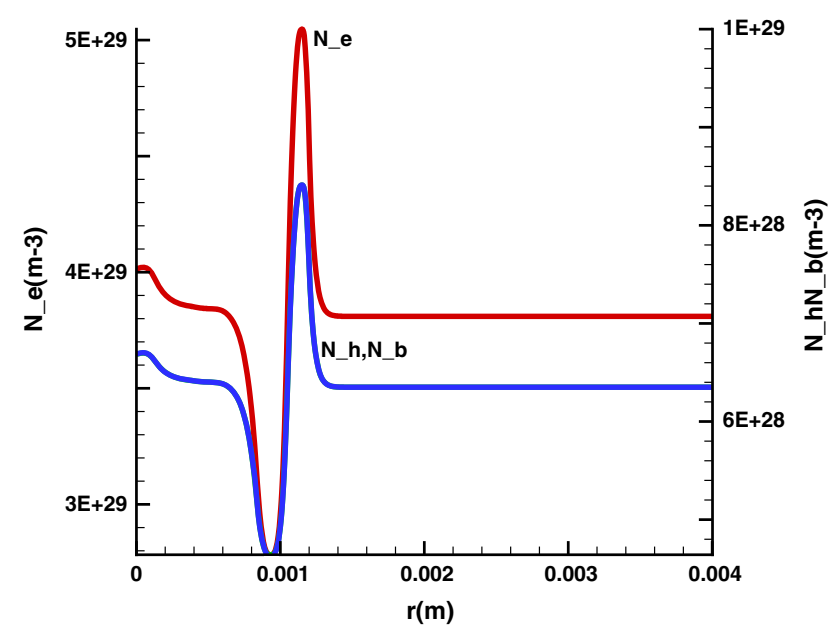

Fig. 2 Solid HB11 cylinder of $5 \mathrm{~mm}$ radius irradiated by a ps-10 20 $\mathrm{W} / \mathrm{cm}^{2}$ laser pulse of $248 \mathrm{~nm}$ wavelength at concentric radius of $1 \mathrm{~mm}$. After $100 \mathrm{ps}$, the dependence on the radius $\mathrm{r}$ is shown for the density of electrons $\mathrm{N}_{\mathrm{e}}$, boron $\mathrm{N}_{\mathrm{b}}$ and hydrogen $\mathrm{N}_{\mathrm{h}}$ (sequence at $\mathrm{r}=0$ from above) using a 10 kilotesla magnetic field 


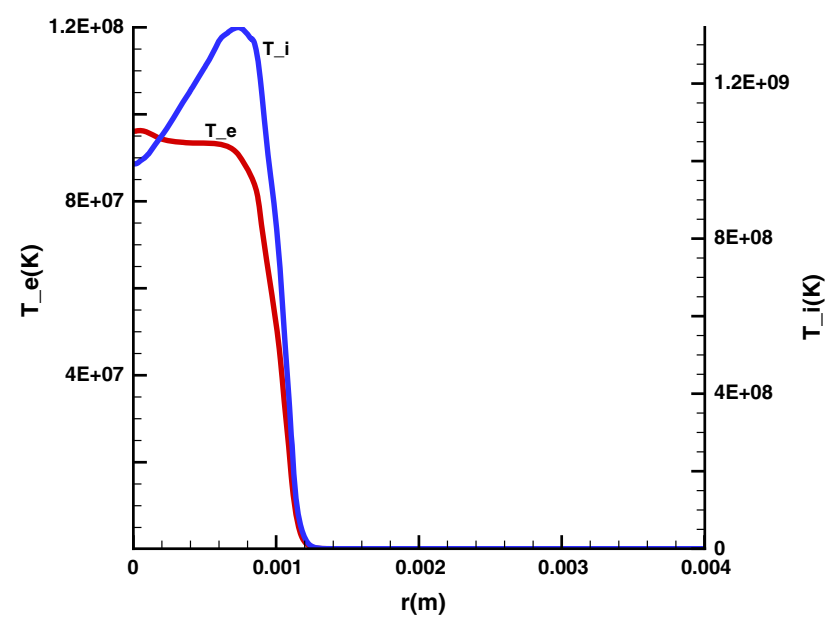

Fig. 3 Same as Fig. 2 with electron and ion temperature

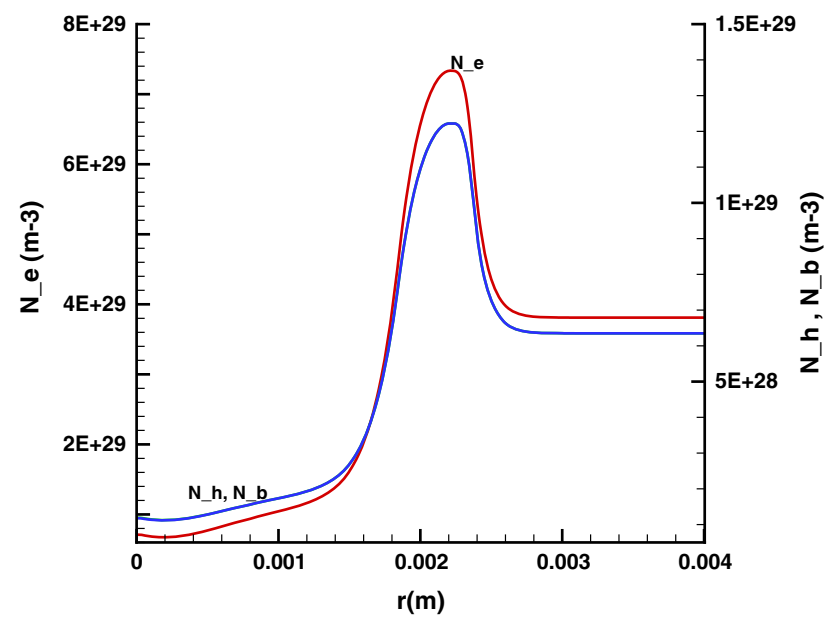

Fig. 4 Same as Fig. 2 after 1,000 ps

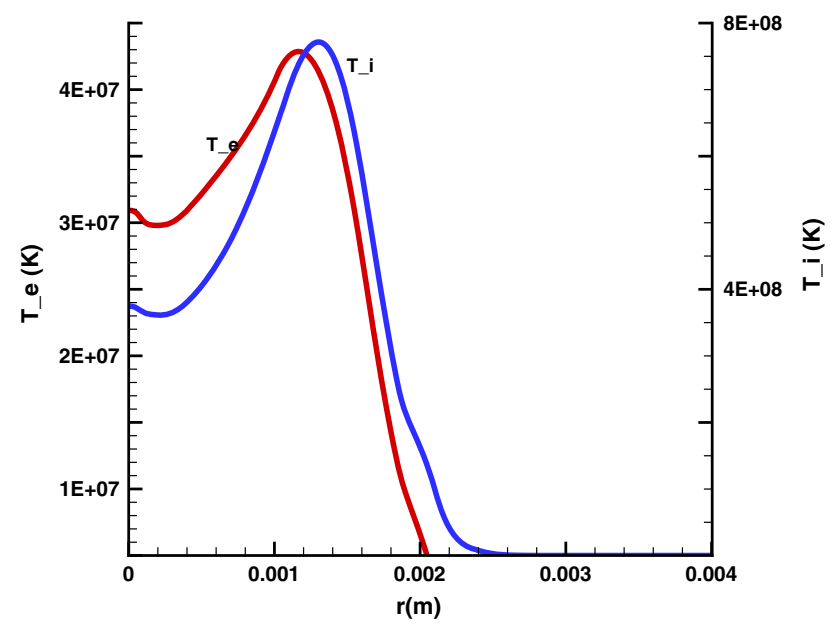

Fig. 5 Same as Fig. 4 with electron and ion temperature

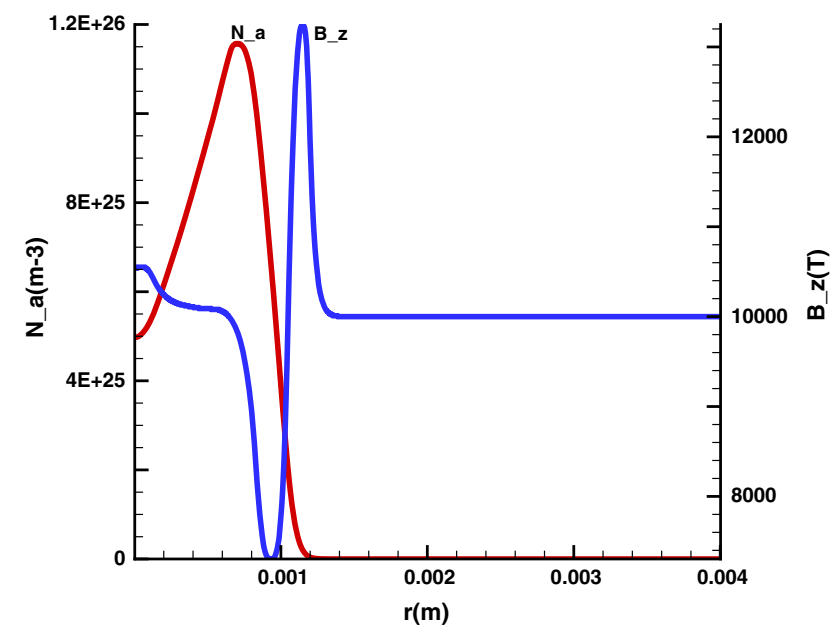

Fig. 7 Same case as Fig. 2: solid HB11 cylinder of $5 \mathrm{~mm}$ radius irradiated by $\mathrm{ps}-10^{20} \mathrm{~W} / \mathrm{cm}^{2}$ laser pulse of $248 \mathrm{~nm}$ wavelength. Radial density at $100 \mathrm{ps}$ of primary produced alpha particles ending at $1 \mathrm{~mm}$ laser irradiation radius and the magnetic field strength depending on the radius $\mathrm{r}$ (in untouched fuel 10 kilotesla at radius above $1 \mathrm{~mm}$ )

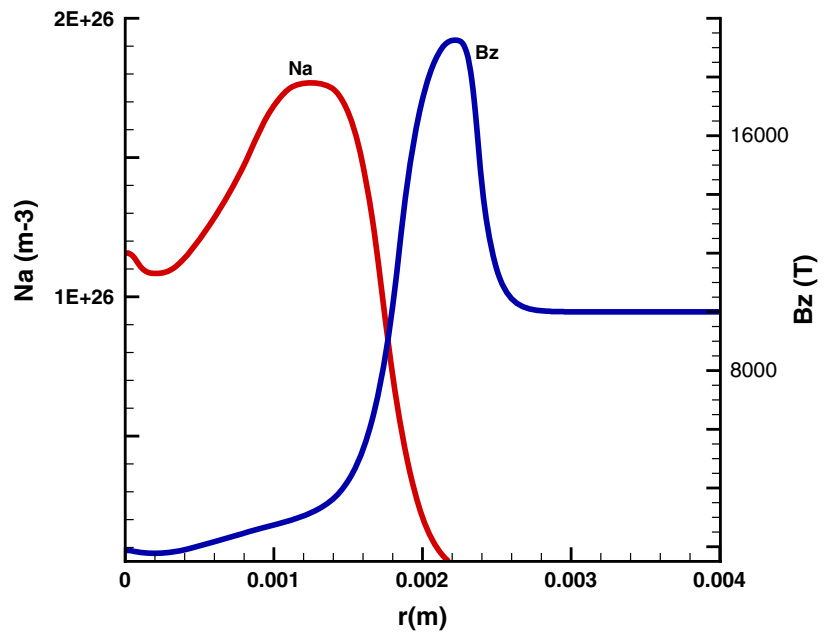

Fig. 8 Same as Fig. 7 at 1,000 ps 